\title{
Improving Participation of Users in Coastal Web Atlases
}

\author{
Kathrin Kopke, CMRC, University College Cork, Ireland, k.kopke@ucc.ie \\ Ned Dwyer, CMRC, University College Cork, Ireland, n.dwyer@ucc.ie \\ Kathy Belpaeme, Coordination Centre for ICZM, Belgium, kathy.belpaeme@kustbeheer.be \\ Marcia Berman, Virginia Institute of Marine Science, USA, marcia@sweethall.wetlan.vims.edu \\ Kathy Taylor, Washington Department of Ecology, USA, kathy.taylor@ECY.WA.GOV \\ David Hart, Wisconsin Sea Grant, University of Wisconsin, USA, dahart@wisc.edu \\ Dawn Wright, Oregon State University, USA, dawn@dusk.geo.orst.edu
}

\begin{abstract}
Coastal mapping plays an important role in informing decision makers on issues such as national sovereignty, resource management, maritime safety and hazard assessment. A key aspect of this trend has been the development of coastal web atlases (CWAs), based on web enabled geographic information systems (GIS). CWAs contain spatial data and thematic information on environmental, social and economic aspects of the coast and provide an integrated visualisation, download and sometimes analysis environment.
\end{abstract}

An important element of CWA development is to ensure that the needs of the target audience are being addressed. Sometimes there can be a break in developer-user communication after the initial atlas design phase is completed. Identifying how to maintain effective engagement by CWA providers with end users is a key task for development teams. A key initial task is to profile the target end users. Workshops and surveys can be effective at this stage. Launch events and media exposure is used effectively to build user communities, however usage drops when such publicity events are discontinued. During the atlas operations phase baseline web statistics are often gathered, but these give a somewhat limited idea of the end user community and their use of the atlases.

Additional means of remaining in touch with users and incorporating their requirements in CWAs need to be used. Web 2.0 applications such as "like/dislike" buttons and content comment boxes are used with success in social networking and some news media sites and their utility should be considered for CWAs. Focus groups have also been used with success to complete semi-structured surveys when trying to gather answers to specific questions. It is important to demonstrate to users that the survey has a goal such as atlas improvements in order to motivate them to provide information.

Enhancing users' sense of ownership achieved by web applications that allow more direct user input has been accomplished by some CWAs. The strengths and weaknesses of such an approach should be further explored by individual CWAs, as this could enhance the understanding of user needs and improve the relationship of the atlas developer to the user community. Atlas developers within the International Coastal Atlas Network (ICAN) who have worked on the Marine Irish Digital Atlas, the Belgian Coastal Atlas, Chesapeake Bay Atlases, the Washington Coastal Atlas and the Wisconsin Coastal Atlas have significant experience in gathering and analysing user feedback and are investigating means of enhancing their interaction with the end-user community.

Keywords: coastal web atlas; International Coastal Atlas Network; coastal management; enduser participation, web GIS. 


\subsection{Introduction}

The use of web-enabled geographical information systems (GIS) to develop continent-wide, national, regional or local coastal web atlases (CWA) has grown considerably worldwide over recent years (Dwyer and Wright, 2008). Such developments have led to the emergence of the International Coastal Atlas Network (ICAN), which to date consists of 35 organisations from 10 countries. ICAN defines a CWA as: "...as a collection of digital maps and datasets with supplementary tables, illustrations, and information that systematically illustrate the coast, oftentimes with cartographic and decision support tools." (Wright et al., 2010a).

ICAN has grown over the past five years from an idea to a more formal virtual community of practice, as the need for capacity building and support via a more established organization was expressed by participating organisations (Dwyer and Wright, 2008). The network has captured the interest of local and state governments, non-governmental organizations, universities and internationally well-known organisations such as the National Oceanic and Atmospheric Administration (NOAA) in the United States and the European Environment Agency (Dwyer and Wright, 2008). Such interest is not surprising as CWAs have the potential to address the needs of very diverse target audiences or end-user groups by providing fundamental information to aid regional decision- and policy-making, covering themes such as coastal recreation, governance, hazard mitigation, climate change impacts and vulnerability mapping, coastal management and marine spatial planning, and many more (Wright et al., 2010b). Indeed, recognizing the educational and informational role of such atlases, the European Commission has launched a pilot version of its European Atlas of the Seas (European Commission, 2010).

Target audience or end user group input, ideally as part of a user-centred design approach, during development of any web-based application is crucial as such input ensures that the resource will meet their needs (Dwyer et al., 2010; Nyerges et al., 2010). However, analyses of recurrent user feedback followed by appropriate CWA update is as important to keep the resource up to date and attractive for the intended target audience (Wright et al., 2010b). Within the ICAN network CWAs have been shown to have common as well as varying approaches when trying to address the needs of their end user groups. This paper looks at a number of approaches used to engage end users and discusses their relative advantages and disadvantages. It then proposes additional new approaches that should be investigated by CWA developers to ensure better engagement with their end-user community.

\subsection{End-user groups}

Two of the most important questions for any CWA developer from the outset should be, "who is going to use the resource"closely followed by "how can this CWA facilitate and support my intended end user group(s)?" (Dwyer et al., 2010; Nyerges et al., 2010; Wright et al., 2010b). This section illustrates how atlas developers often focus their resource on different end-user groups. This impacts on both the content of and the design and interface of the CWA.

One central goal in the development of the Marine Irish Digital Atlas (MIDA: http://mida.ucc.ie) was to cater to a broad audience, so that the casual visitor as well as students and professionals could utilise this resource when searching for coastal and marine data and information (Dwyer et al., 2010). One approach to facilitating this was the incorporation of distinct search options that allowed general and specific data searches. In addition a MIDA tutorial was provided to introduce fist time visitors to the website and explain the range of uses. The MIDA is an island-wide initiative transcending cross-border interests between the Republic and Northern Ireland. This is of great interest to others facing the challenge of a multi-national atlas (e.g. Caribbean Marine Atlas group) (Dwyer et al., 2010). 
The purpose of the Washington Coastal Atlas (WCA:

http://www.ecy.wa.gov/programs/sea/sma/atlas_home.html) is to make relevant information easily available for use in coastal and shoreline planning and management. Consequently, the primary target audiences are (1) local governments implementing Washington's Coastal Zone Management Program through local Shoreline Master Programs and (2) state government agencies with authority to regulate or manage activities on the shorelines and tidelands of Washington. Other important user groups include Native American Tribal governments, Federal government agencies, outdoor recreation groups, non-governmental advocacy organisations, and private citizens. In addition to physical, biological, regulatory, and jurisdictional data layers, the WCA also provides a time series of oblique aerial photos of Washington's entire marine shoreline. These photos are accessed by a much wider audience than the primary target. Furthermore, the WCA serves land cover change data available from NOAA, which has a more specialized user group. In order to continue to serve the primary target audience well, while increasing ease of use for other users who may come to the WCA looking for only one type of data (such as shoreline photos) the WCA is in the process of adding new information as well as carrying out a major redesign and upgrade with a strong focus on redesigning the user interface to allow easy access to specific data sets, as well as to multiple data layers.

The Chesapeake Bay, the largest estuary in the United States, includes two CWAs developed by the bordering states of Virginia and Maryland. Virginia's Geospatial and Educational Mapping System (GEMS: http://www.deq.virginia.gov/coastal/coastalgems.html ) brings together over 50 different data layers with a focus on coastal planning and conservation. Maryland's Shorelines Online (MSO: http://shorelines.dnr.state.md.us/) combines map information and links to data with outreach and educational information via a structured web interface (Berman and McCall, 2010). Particular areas of interest are highlighted for specified end users including coastal managers, property owners, marine contractors and teachers (Berman and McCall, 2010). The MSO is undergoing extensive revision at this time which will reflect a change in format as well as expanded coverage to the open ocean coast.

Currently under development, the Wisconsin Coastal Atlas which is targeting coastal resource managers aims to construct a geoportal for coastal maps and data, provide a platform for the development of spatial decision support tools, and promote interoperable state and local spatial data as the foundation of a Great Lakes spatial data infrastructure (Nyerges et al., 2010). The University of Wisconsin Sea Grant Institute and its partners created a LOGIC Model as part of a structured process to build the atlas. A LOGIC Model integrates project design and evaluation and documents project goals, objectives, activities, outputs, and outcomes (Mayeske et al., 2001; McLaughlin and Jordan, 1999). The project team requested assistance from staff of the NOAA Coastal Services Center to learn how to apply a LOGIC Model to a CWA project and guide drafting the initial version of the LOGIC Model.

The Belgian Coastal Atlas (http://kustatlas.be) targets a broad audience including teachers, scientists, civil servants and politicians (Belpaeme and Maelfait, 2010). The successful publication of a hardcopy coastal atlas led to the development of an online version after numerous requests for such a resource were received by the Coordination Centre for ICZM. This online version of the hardcopy atlas has additional features to allow download and printing of maps. In addition the website is available in four languages and therefore easy accessible for an international audience. A fully web-enabled version of the Atlas is currently under development (Belpaeme and Maelfait, 2010). 


\subsection{Workshops}

Many CWA developers use workshops to gather user requirements and gain user feedback both during the development phase of the resource and later to keep the resource aligned with user wishes. Furthermore workshops also provide a platform to raise awareness of new CWAs and their uses as well as to exchange information with other CWA developers (Wright et al., 2010b).

The MIDA team targeted some key user groups and potential data contributors in workshops early in the Atlas development cycle. The workshop focused on interaction with a prototype (Dwyer et al., 2010). The prototype was very effective in stimulating discussion on user likes and dislikes and consolidation of the comments and feedback allowed design changes for the final version of the atlas. When this was released three workshops in different locations around Ireland were held with the aim of advertising the Atlas and gathering additional feedback. This feedback addressed ongoing developments of the resource and highlighted interest in specific additional themes for which users wanted spatial data. To date MIDA contains spatial data and additional information for over $85 \%$ of those themes suggested by workshop participants (Dwyer et al., 2010).

The Washington Coastal Atlas (WCA) team utilised and organised the West Coast Coastal Atlas workshop to increase contact among existing and emerging coastal web atlas efforts on the US west coast in order to increase information flow and opportunities for collaboration on CWA projects (Wright et al., 2010b). The workshop proved to very useful for the WCA team as specific feedback from other CWA developers was given. Such peer review was deemed important and could be extended to address issues of coastal data quality and standards (Wright et al., 2010b).

Developers of Virginia's Coastal GEMS hosted workshops at the time the site was launched to build awareness, encourage use, and provide training. As part of that series, user representatives provided feedback through a written survey. Maryland also uses workshops as the means to encourage use and train end users. Feedback is welcomed, but no official survey or comments are necessarily solicited.

As the Belgian Coastal Atlas was initially merely a transition from the book version towards an on-line version, end-users were not involved. However, in 2009 the team started developing towards a fully web-enabled version with a high level of interactivity in the mapping tool. To ascertain end-user needs a workshop was organised in April 2009, to help determine how the web atlas would be developed. A main conclusion of the workshop was that end-users see an important role for the coastal atlas as a communication tool and a portal for coastal information. Providing unique and integrated maps is seen as an important added value.

Feedback also reaches the Belgian team during or in relation to related workshops and conferences, and also spontaneous comment is received regularly from a broad range of atlas users. All comments and questions are registered and monitored.

\subsection{Publicity}

Publicity is the lifeline of any CWA in order to raise awareness as well as to attract new users (Dwyer et al., 2010). Participating in ICAN alone provides constant exposure to an international audience for any CWA (Dwyer et al., 2010), as ICAN is regularly presented at a variety of conferences and specialist meetings worldwide (e.g. GIScience 2008, Littoral 2008, CoastGIS 2009, Coastal GeoTools 2009, Third INSPIRE Conference 2009). 
Initially MIDA was introduced at Irish and international specialist conferences and articles were written for a number of popular publications in the coastal and marine field to present the resource (Dwyer et al., 2010). An Atlas brochure was published and disseminated widely. Finally an official launch event generated significant media interest with publications in the national and regional press, which led to a significant increase in visits to the Atlas during and immediately after the publicity events (Dwyer et al., 2010).

The Belgium Coastal Atlas also held a very successful launch event, which resulted in a unique visitor count of almost 5000 shortly after the launch (Kopke and Belpaeme, 2008). The Atlas also used a set of postcards with aerial photographs which were available for purchase in book shops at the coast. These were very effective as promotional gifts and helped to raise further awareness (Kopke and Belpaeme, 2008). The team in Belgium also takes every opportunity to give demonstrations on the atlas during events for specific target groups, such as geography teachers, coastal stakeholders and scientists.

Local venues, conferences and workshops present opportunities to demonstrate productsand new technologies. The Virginia Coastal GEMS and the Washington Coastal Atlas have been presented and demonstrated at several venues that target the anticipated user community. These may not be venues typically attended by CWA developers, but rather the local planners who represent the majority of the target audience. This has helped build awareness of these applications and has led to enhanced use. The Washington Coastal Atlas has also been presented at inter and intra-agency coordination meetings among state natural resource agencies.

Other approaches to publicity can also be effective. CWAs can be featured on other hightraffic web sites as a "site of the month" (such as the Open Geospatial Consortium Website of the month within their virtual "press room" at www.opengeospatial.org). Recently ICAN participated in the webinar series of NatureServe's rapidly-growing Ecosystem Based Management Tools Network (ebmtools.org), which helped to increase further the international visibility of the organisation as well as specific, user-centred features of MIDA and the Oregon Coastal Atlas.

Completion of a major upgrade or redesign effort or the addition of an important new data set allows the opportunity to engage the media. The new version of the Washington Coastal Atlas will be launched in Autumn 2010 and the Washington Department of Ecology is planning a press release and a short video to be posted on the agency's web site.

\subsection{Web statistics and surveys}

Web statistics allow CWA developers to gauge trends and updates by providing information on data downloads, number of visitors and country code and give some basic easily accessible information concerning the make-up of the CWA end user community. Although such information is very useful as it provides fast but somewhat general end-user feedback it does not allow detailed qualitative analyses (Wright et al., 2010b).

MIDA user statistics for example confirm that most Atlas visitors come from the Republic of Ireland, followed by visitors from the UK and the USA. The web statistics also showed the most popular downloadable data layers are from the socio-economic activity section, specifically water-based recreation. Similarly the Belgium Coastal Atlas is using such statistics to confirm visitor numbers as well as finding that most end users are utilising the resource during the week in work hours indicating work related queries (Belpaeme and Maelfait, 2010). 
In 2009, ICAN launched a user focussed web-based survey so developers could improve CWA products and content. The survey was developed by a collective group of ICAN members who represented the CWA developers, data providers, program managers, and end users. The survey used the Survey Monkey tool and could be accessed only through the ICAN website. Through their own CWAs, ICAN members encouraged their respective national and state CWA users to visit the site. The survey was not well received and participation was extremely low. This was most likely a function of visitors to the ICAN website in general and knowledge that the survey existed. However, later discussions within the ICAN community suggested the survey was difficult to find and the format may have been too cumbersome.

In the revised version of the Belgian Coastal Atlas, a discretionary feedback form will be integrated in the site. Questions will be asked about the quality of the site, data content, its success in meeting user needs, etc. Completion of such a form will allow profiling of the user community. However, before implementing such a feedback mechanism, discussions with representative users will take place to determine the most appropriate content and approaches to take to maximise uptake of the form.

\subsection{New approaches to engaging users}

While all of the above mentioned mechanisms to engage end-users and gather feedback have proven to be useful for many CWAs, others have not worked as intended. User surveys are often seen as tedious and unless users see that action is taken in regard to their feedback they become quickly disillusioned. Most atlases also have "Contact us" links but these tend to be used less than anticipated. For example, there is a specific email address (mida@ucc.ie) to contact the MIDA team however this is used quite infrequently with most emails being data requests rather than comment or feedback on existing content or use. (Dwyer et al., 2010).

New opportunities to obtain feedback and engage the end user have to be considered. Web 2.0 applications offer some interesting possibilities. "Like/dislike" buttons and comment features could be associated with specific layers, maps or thematic content allowing users to post immediate feedback, without the need to look for particular contact points via other links within the web site. These approaches are used on social networking and many news media sites and appear to engage users more readily. Some of NASA's news websites have begun incorporating such applications (e.g. http://www.nasa.gov/news/index.html). However, such features need to be monitored carefully and may need a moderator to avoid inappropriate or misuse, which would also require additional resources and therefore funding.

Training end users to utilise the functional and operative tools in a CWA is often overlooked. Developers and program managers should not assume the end-user possesses the skills necessary to navigate a CWA. The more sophisticated these products become, the more challenging this will be for the audience. To that end, training the end-user should become an integral component in the CWA planning process. The NOAA Coastal Zone Management Program in Virginia, which supports the development of Virginia's Coastal GEMS Atlas, also provides resources to support individualized training on request. While this may not be feasible for CWAs with a national focus, the message here is to remember developers must build the capacity for the end user to become efficient in the use of the atlas through training or risk that the atlas will not be widely used.

Currently CWAs tend to be uni-directional with data, information and maps being delivered from the atlas developers to the users. By giving users new possibilities to create their own map or introduce their own data or information as well as being able to tailor the web atlas for their specific needs, they are more likely to increase usage of the CWA but also contact the Atlas 
managers when specific information or maps are lacking. Sites such as www.openstreetmap.org illustrate how a very disparate community can build a map and that self policing tends to ensure that the information is correct.

\section{Conclusions}

CWA development is often driven by technical innovation and even though the importance of the end user has been recognised, the ability to engage them actively over the lifetime of an atlas has not been particularly successful. Profiling users and their use of an atlas in itself is a challenge as baseline web statistics are only partially successful in this regard. Pop-up windows encouraging users to identify themselves or carry out an online survey are perceived to be annoying and can ultimately lead to users abandoning a site, so more subtle and innovative ways need to be considered. Mechanisms that have been shown to be popular and successful in other settings should be considered such as those utilised in web 2.0 applications such as "like/dislike" buttons and comment cards. These tools might not provide a detailed user evaluation of the CWA but can indicate trends and offer instant impressions of user opinion on information, format or layers in a CWA.

User surveys are often used with success when gathering user requirements during CWA development. When used during workshops and for targeted individuals or organisations, they are extremely effective. However, their use during the operational and maintenance phase of a CWA needs careful consideration if they are to be of value. Focus groups could be targeted for more detailed semi-structured surveys to answer specific questions or the CWA can be demonstrated and feedback solicited during related events as has been the case with the Virginia Coastal GEMS and the Washington Coastal Atlas Enhancing users' sense of ownership such as that achieved by web applications that allow more direct user input and even provision of data should be further explored by individual $7 \mathrm{CWAs}$, as this could deepen the understanding of user needs and improve the relationship of the atlas developer with the user community. However, issues of data and information quality control as well as access rights to the atlases and security concerns would have to be addressed, and these differ depending on the type of CWA host organisation.

\section{References}

Belpaeme, K. and Maelfait, H. 2010. Belgium. In D. Wright, E. Dwyer and V. Cummins (Eds.), Coastal Informatics: Web Atlas Design and Implementation (pp. 156-164). Information Science Reference, IGI Global, ISBN10: 1615208151.

Berman, M. and McCall, C., 2010. Virginia and Maryland, USA. In D. Wright, E. Dwyer and V. Cummins (Eds.), Coastal Informatics: Web Atlas Design and Implementation (pp. 131-144). Information Science Reference, IGI Global, ISBN10: 1615208151.

Dwyer, E., Kopke, K., Cummins, V., O'Dea, E. and Dunne, D., 2010. Ireland. In D. Wright, E.Dwyer and V. Cummins (Eds.), Coastal Informatics: Web Atlas Design and Implementation (pp. 105-130). Information Science Reference, IGI Global, ISBN10: 1615208151.

Dwyer, N. and Wright, D.J., 2008. Report of International Coastal Atlas Network Workshop 3 on Federated Coastal Atlases: Building on the Interoperable Approach. European Environment Agency, Copenhagen, Denmark, 65 pages.

Mayeske, G. W. and Lambur, M. T., 2001. How to Design Better Programs: A Staff Centered Stakeholder Approach to Program Logic Modeling. Crofton, MD: The Program Design Institute. 
McLaughlin, J. A., and Jordan, G. B., 1999. Logic models: A tool for telling your program's performance story, Evaluation and Program Planning. 22(1), 65-72.

Nyerges, T., Belpaeme, K., Haddad, T. and Hart D., 2010. Creating a Usable Atlas. In D.

Wright, E. Dwyer and V. Cummins (Eds.), Coastal Informatics: Web Atlas Design and Implementation (pp. 256-266). Information Science Reference, IGI Global, ISBN10: 1615208151.

Kopke, K. and Belpaeme, K., 2008. Atlas User Expectations and Experiences at LITTORAL 2008: A Changing Coast - Challenge for the Environmental Policies, Venice, Italy.

Wright, D., Dwyer N. and Cummins, V., 2010a. Introduction. In D. Wright, E. Dwyer and V. Cummins (Eds.), Coastal Informatics: Web Atlas Design and Implementation (pp.1-11). Information Science Reference, IGI Global, ISBN10: 1615208151.

Wright, D., Dwyer, N., Kopke, K., and O'Dea, L., 2010b. Report of International Coastal Atlas Network Workshop 4: Formalizing the Network, Engaging the Mediterranean. UNESCO International Centre for Theoretical Physics, Trieste, Italy, 16-20 November 2009, ICAN online, 79 pages. 8 